ORIGINAL ARTICLE

\title{
Prednisolone or Pentoxifylline for Alcoholic Hepatitis
}

\begin{abstract}
Mark R. Thursz, M.D., Paul Richardson, M.D., Michael Allison, Ph.D., Andrew Austin, M.D., Megan Bowers, M.Sc., Christopher P. Day, M.D., Ph.D., Nichola Downs, P.G. Cert., Dermot Gleeson, M.D., Alastair MacGilchrist, M.D., Allister Grant, Ph.D., Steven Hood, M.D., Steven Masson, M.A., Anne McCune, M.D., Jane Mellor, M.Sc., John O'Grady, M.D., David Patch, M.D., Ian Ratcliffe, M.Sc., Paul Roderick, Ph.D., Louise Stanton, M.Sc., Nikhil Vergis, M.B., B.S., Mark Wright, Ph.D., Stephen Ryder, D.M., and Ewan H. Forrest, M.D., for the STOPAH Trial*
\end{abstract}

ABSTRACT

\section{BACKGROUND}

Alcoholic hepatitis is a clinical syndrome characterized by jaundice and liver impairment that occurs in patients with a history of heavy and prolonged alcohol use. The short-term mortality among patients with severe disease exceeds 30\%. Prednisolone and pentoxifylline are both recommended for the treatment of severe alcoholic hepatitis, but uncertainty about their benefit persists.

\section{METHODS}

We conducted a multicenter, double-blind, randomized trial with a 2-by-2 factorial design to evaluate the effect of treatment with prednisolone or pentoxifylline. The primary end point was mortality at 28 days. Secondary end points included death or liver transplantation at 90 days and at 1 year. Patients with a clinical diagnosis of alcoholic hepatitis and severe disease were randomly assigned to one of four groups: a group that received a pentoxifylline-matched placebo and a prednisolone-matched placebo, a group that received prednisolone and a pentoxifylline-matched placebo, a group that received pentoxifylline and a prednisolone-matched placebo, or a group that received both prednisolone and pentoxifylline.

RESULTS

A total of 1103 patients underwent randomization, and data from 1053 were available for the primary end-point analysis. Mortality at 28 days was 17\% (45 of 269 patients) in the placebo-placebo group, 14\% (38 of 266 patients) in the prednisolone-placebo group, 19\% (50 of 258 patients) in the pentoxifylline-placebo group, and 13\% (35 of 260 patients) in the prednisolone-pentoxifylline group. The odds ratio for 28-day mortality with pentoxifylline was 1.07 (95\% confidence interval [CI], 0.77 to 1.49; $\mathrm{P}=0.69)$, and that with prednisolone was 0.72 (95\% CI, 0.52 to $1.01 ; \mathrm{P}=0.06)$. At 90 days and at 1 year, there were no significant between-group differences. Serious infections occurred in $13 \%$ of the patients treated with prednisolone versus $7 \%$ of those who did not receive prednisolone $(\mathrm{P}=0.002)$.

\section{CONCLUSIONS}

Pentoxifylline did not improve survival in patients with alcoholic hepatitis. Prednisolone was associated with a reduction in 28-day mortality that did not reach significance and with no improvement in outcomes at 90 days or 1 year. (Funded by the National Institute for Health Research Health Technology Assessment program; STOPAH EudraCT number, 2009-013897-42, and Current Controlled Trials number, ISRCTN88782125.)
The authors' affiliations are listed in the Appendix. Address reprint requests to Dr. Thursz at the Hepatology Section, Imperial College, Norfolk PI., London W21NY, United Kingdom, or at m.thursz@ imperial.ac.uk.

*A complete list of the investigators in the Steroids or Pentoxifylline for Alcoholic Hepatitis (STOPAH) trial is provided in the Supplementary Appendix, available at NEJM.org.

N Engl J Med 2015;372:1619-28. DOI: $10.1056 /$ NEJMoal412278 Copyright (C) 2015 Massachusetts Medical Society. 


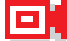

A Quick Take summary is available at NEJM.org

\section{A} LCOHOLIC HEPATITIS IS A DISTINCT manifestation of alcoholic liver disease that is characterized by jaundice and liver failure. This condition develops in persons with a history of prolonged and heavy alcohol use. ${ }^{1}$ The severity of alcoholic hepatitis is conventionally defined by Maddrey's discriminant function, which is calculated as $4.6 \times$ (patient's prothrombin time in seconds-control's prothrombin time in seconds) + patient's serum bilirubin level in milligrams per deciliter; a value of 32 or higher indicates severe alcoholic hepatitis that carries an adverse prognosis, with mortality of 20 to $30 \%$ within 1 month after presentation and 30 to $40 \%$ within 6 months after presentation. ${ }^{2}$

A number of therapies have been evaluated for the treatment of alcoholic hepatitis, but only two drugs have been incorporated into the treatment guidelines published by the American Association for the Study of Liver Disease and the European Association for the Study of the Liver ${ }^{3,4}$. In a 2008 Cochrane meta-analysis of 15 randomized trials published since 1971 that compared glucocorticoids with placebo or no intervention, Rambaldi et al. investigated the role of glucocorticoid therapy for this condition. ${ }^{5}$ Despite this apparent wealth of evidence, controversy persists. Advocates of the treatment cite significant reduction in short-term mortality, whereas detractors raise questions about the risks of sepsis and gastrointestinal hemorrhage with glucocorticoid therapy. In the largest placebo-controlled study to date, investigators treated 90 patients with prednisolone and found no benefit of that therapy over placebo administered in a similar group of patients. ${ }^{6}$ This study was hampered by the inclusion of patients with moderate or severe alcoholic hepatitis and those with alcohol-related cirrhosis. In the only study we found that required histologic confirmation of alcoholic hepatitis in all patients, prednisolone was associated with a short-term reduction in mortality, but this benefit was not apparent after 2 years. ${ }^{7,8}$ The systematic review by the Cochrane group revealed a trend toward a benefit with glucocorticoids that was not statistically significant. ${ }^{5}$ However a reanalysis of the five largest studies indicated a significant benefit from glucocorticoids; in this meta-analysis, 28-day mortality among patients with discriminant function scores of 32 or higher was 20\% among those who were treated with prednisolone, as compared with $34 \%$ among those who received placebo $(\mathrm{P}<0.001) .{ }^{9}$
Among four randomized, controlled trials in which pentoxifylline was compared with placebo for the treatment of alcoholic hepatitis, one showed a significant benefit. ${ }^{10}$ All 100 patients enrolled had a discriminant function that was greater than 32. The mortality was $24.6 \%$ in the pentoxifylline group as compared with $46.1 \%$ in the placebo group ( $\mathrm{P}=0.04)$. The principal benefit related to pentoxifylline appeared to be a reduction in the number of deaths attributed to the hepatorenal syndrome. However, two meta-analyses have not shown any convincing benefit associated with pentoxifylline..$^{11,12}$

Two small studies have compared glucocorticoids with pentoxifylline, but the results were inconsistent. ${ }^{13,14}$ Two other studies have compared the effect of glucocorticoid monotherapy with that of combined treatment with glucocorticoids and pentoxifylline but showed no benefit from the addition of pentoxifylline. ${ }^{15,16}$ The purpose of our trial - Steroids or Pentoxifylline for Alcoholic Hepatitis (STOPAH) - was to determine whether prednisolone or pentoxifylline administered for a 28-day period reduced short-term and medium-term mortality among patients admitted to a hospital with severe alcoholic hepatitis.

METHODS

\section{STUDY DESIGN AND OVERSIGHT}

The design and rationale for the trial have been described previously. ${ }^{17}$ STOPAH was a multicenter, randomized, double-blind trial with a 2-by-2 factorial design that was intended to evaluate the treatment effect of prednisolone and of pentoxifylline in the management of severe alcoholic hepatitis. A trial-management group designed the study (see the protocol, available with the full text of this article at NEJM.org). The study was approved by the Multicenter Research Ethics Committee (reference number 09/MRE09/59), and clinical trial authorization was received from the Medicines and Healthcare Products Regulatory Agency. Written informed consent was obtained from each patient or from his or her legal representative until such time as the patient recovered mental capacity. The trial was conducted and reported with fidelity to the protocol, the Medicines for Human Use (Clinical Trials) Regulations 2004, as amended in 2006, the European Union Clinical Trials Directive (Directive 2001/20/EC) guidelines, the principles of the International Conference on 
Harmonisation Good Clinical Practice under the oversight of University Hospital Southampton NHS Foundation Trust, and the provisions of the Declaration of Helsinki. An independent data monitoring and ethics committee, whose members were aware of the group assignments, was convened to review the conduct of the trial and to analyze primary end-point data, using prespecified stopping guidelines, after the recruitment of 200,400 , and 800 patients, to avoid continued recruitment in the event that a definitive result had been achieved.

Data collected by site investigative teams were submitted to the clinical trials unit and analyzed by study statisticians. The first author wrote the first draft of the manuscript, with substantial contributions from the coauthors. All the authors vouch for the accuracy and completeness of the data and analyses.

\section{PATIENTS}

The trial included patients with a clinical diagnosis of alcoholic hepatitis. Clinical diagnosis was chosen because the use of liver biopsy in this group of patients is uncommon, and the aim was to recruit a large number of participants whose condition would reflect as closely as possible the condition of patients seen in clinical practice. ${ }^{18,19}$ Patients were recruited from January 2011 through February 2014 at 65 hospitals across the United Kingdom. All patients admitted with suspected severe alcoholic hepatitis were evaluated for eligibility. A clinical diagnosis that was based on a history of recent excess alcohol consumption and the absence of other causes of liver disease was used for trial recruitment. Inclusion criteria were an age of 18 years or older, a clinical diagnosis of alcoholic hepatitis, an average alcohol consumption of more than $80 \mathrm{~g}$ per day for men and more than $60 \mathrm{~g}$ per day for women, a serum bilirubin level greater than $80 \mu \mathrm{mol}$ per liter $(4.7 \mathrm{mg}$ per deciliter), and a discriminant function of 32 or higher. Key exclusion criteria were jaundice for more than 3 months, cessation of alcohol consumption for more than 2 months before randomization, the presence of other causes of liver disease, a serum aspartate aminotransferase level greater than 500 IU per liter or serum alanine transaminase level greater than 300 IU per liter, and previous entry into the study within the preceding 6 months.

Standard supportive care and nutritional sup- port were given to each patient. The clinician responsible for each patient made the decision regarding other treatments, such as terlipressin for patients in whom hepatorenal failure was developing, acid suppression for prophylaxis against gastrointestinal hemorrhage, antibiotics, and vitamin supplementation. Patients with renal failure (defined as a creatinine level $>500 \mu \mathrm{mol}$ per liter [ $>5.7 \mathrm{mg}$ per deciliter] or the requirement for renal-replacement therapy), active gastrointestinal bleeding, or untreated sepsis, and patients requiring inotropic support with epinephrine or norepinephrine, were excluded unless the condition stabilized within the first 7 days after admission to the hospital.

\section{RANDOMIZATION}

A Web-based computer system (Tenalea, FormsVision) was used to enroll eligible patients and randomly assign them to study groups. The randomization schedule was created with the use of Stata software, version 11 (StataCorp). Randomization was performed with a block size of four, with stratification according to geographic area and risk category. The high-risk category consisted of patients who had had an occurrence of gastrointestinal bleeding, renal impairment, or sepsis before randomization. All other patients were assigned to the intermediate-risk category.

Patients were randomly assigned to one of four groups, with one group receiving a pentoxifyllinematched placebo and a prednisolone-matched placebo, the second group receiving $40 \mathrm{mg}$ of prednisolone daily and a pentoxifylline-matched placebo, the third group receiving $400 \mathrm{mg}$ of pentoxifylline three times daily and prednisolonematched placebo, and the fourth group receiving $40 \mathrm{mg}$ of prednisolone daily and $400 \mathrm{mg}$ of pentoxifylline three times daily. All patients were prescribed treatment for 28 days.

\section{END POINTS}

The primary end point of the trial was mortality at 28 days. Secondary end points included mortality or liver transplantation at 90 days and at 1 year.

\section{STATISTICAL ANALYSIS}

We estimated that a sample of 513 patients receiving each active agent and an equal number not receiving each agent would be required to detect a reduction in 28-day mortality from 30\% in the latter groups to $21 \%$ in the former groups. 
Thus, in total, our trial would require enrollment of 1026 patients. We allowed for a rate of withdrawal or loss to follow-up of approximately $10 \%$ and therefore aimed to recruit 1200 patients to the study.

The analysis was conducted on an intentionto-treat basis. The comparison of mortality at 28 days between treated and untreated groups was performed with the use of logistic regression, with adjustments for risk category (high or intermediate) and factorial design. Mortality and rates of liver transplantation at 90 days and 1 year were compared with the use of the same strategy. A test for treatment interaction was performed as a secondary analysis. Cox proportional-hazards regression was used to compare 1-year survival among the groups, and Kaplan-Meier curves for 1-year survival were plotted. All P values were two-sided.

A univariate logistic-regression analysis was performed for the end points of 28-day mortality, 90-day mortality, and 1-year mortality. Separate models were fitted for conventional prognostic scores (discriminant function, Model for End-Stage Liver Disease score, Glasgow alcoholic hepatitis score, and Lille score) and for clinical and laboratory variables, to investigate whether they were significant predictors of mortality. As part of a prespecified analysis, individual and treatment variables that were found to be significant in univariate analyses were used in multivariate logisticregression analysis, and backward elimination (which was not prespecified) was applied at a 5\% significance level.

\section{RESULTS}

\section{PATIENTS}

Over a 3-year period, 5234 patients were screened, and after the application of eligibility criteria, 1103 patients were randomly assigned to one of the four treatment groups: 276 to the placebo-placebo group, 277 to the prednisolone-placebo group, 276 to the pentoxifylline-placebo group, and 274 to the prednisolone-pentoxifylline group (see Fig. S1 in the Supplementary Appendix, available at NEJM.org). All the patients were followed for 12 months or until the time of their death, with the exception of patients enrolled at the end of the trial. Owing to limitations on funding, the trial was stopped after all enrolled patients had completed at least 28 days of follow-up. At the time the trial was stopped, 33 patients who underwent randomization during the last 90 days of the trial could not be included in the 90-day or 12-month analyses. In addition, there were 159 patients who underwent randomization within 90 days to 12 months before the end of trial who could not be included in 12-month analyses. The four groups were well matched with regard to their baseline characteristics, including laboratory values (Table 1). At 28 days, $16 \%$ of the patients had died, $1 \%$ had been lost to follow-up, and $2 \%$ had withdrawn from the study. At 90 days, $29 \%$ of the patients (285 of 968 patients) had died, 5\% had been lost to follow-up, 3\% had withdrawn, and 4\% had not completed follow-up owing to cessation of the study. At 1 year, $56 \%$ of the patients ( 421 of 747 patients) had died or undergone liver transplantation (3 patients), $8 \%$ had been lost to followup, $4 \%$ had withdrawn, and $20 \%$ had not completed follow-up owing to cessation of the study.

\section{END POINTS}

At 28 days, 45 of 269 patients (17\%) in the placebo-placebo group had died, 38 of 266 patients $(14 \%)$ in the prednisolone-placebo group had died, 50 of 258 patients (19\%) in the pentoxifyllineplacebo group had died, and 35 of 260 patients $(13 \%)$ in the prednisolone-pentoxifylline group had died. There was no significant treatment interaction between prednisolone and pentoxifylline $(\mathrm{P}=0.41)$. In the prespecified analysis of the primary outcome (a logistic-regression analysis that was adjusted for the risk category [high or intermediate] used in the randomization and for the factorial design), the odds ratio for 28-day mortality among patients who received pentoxifylline (those in the pentoxifylline-placebo group or those in the prednisolone-pentoxifylline group), as compared with patients who did not receive pentoxifylline was 1.07 ( $95 \%$ confidence interval [CI], 0.77 to $1.49 ; \mathrm{P}=0.69$ ), and the odds ratio among patients who received prednisolone (those in the prednisolone-placebo group or those in the prednisolone-pentoxifylline group), as compared with patients who did not receive prednisolone, was 0.72 (95\% CI, 0.52 to 1.01; $\mathrm{P}=0.06$ ) (Table 2). Neither prednisolone nor pentoxifylline was found to influence mortality or the need for liver transplantation at 90 days or 1 year (Table 2). KaplanMeier curves for survival in each treatment group 
Table 1. Baseline Characteristics of the Patients."

\begin{tabular}{|c|c|c|c|c|c|}
\hline Characteristic & $\begin{array}{l}\text { Placebo- } \\
\text { Placebo } \\
(\mathrm{N}=272)\end{array}$ & $\begin{array}{c}\text { Prednisolone- } \\
\text { Placebo } \\
(\mathrm{N}=\mathbf{2 7 4})\end{array}$ & $\begin{array}{c}\text { Pentoxifylline- } \\
\text { Placebo } \\
(\mathrm{N}=\mathbf{2 7 3})\end{array}$ & $\begin{array}{l}\text { Prednisolone- } \\
\text { Pentoxifylline } \\
(N=273)\end{array}$ & $\begin{array}{c}\text { Total } \\
(\mathrm{N}=1092)\end{array}$ \\
\hline Age $-y r$ & $48.8 \pm 10.3$ & $49.3 \pm 10.6$ & $47.9 \pm 10.2$ & $48.6 \pm 9.8$ & $48.7 \pm 10.2$ \\
\hline Male sex — no. (\%) & $162(60)$ & $177(65)$ & $164(60)$ & $182(67)$ & $685(63)$ \\
\hline \multicolumn{6}{|l|}{ Alcohol consumption - g/day } \\
\hline Women & $153.7 \pm 98.5$ & $141.7 \pm 75.4$ & $145.7 \pm 93.1$ & $157.0 \pm 143.8$ & $149.5 \pm 104.3$ \\
\hline Men & $195.4 \pm 126.5$ & $209.9 \pm 117.7$ & $192.4 \pm 129.8$ & $201.7 \pm 127.3$ & $200.1 \pm 125.2$ \\
\hline $\begin{array}{l}\text { Time from admission to initiation } \\
\text { of treatment — days }\end{array}$ & $6.1 \pm 3.8$ & $6.5 \pm 3.9$ & $6.7 \pm 4.2$ & $6.5 \pm 4.4$ & $6.4 \pm 4.1$ \\
\hline \multicolumn{6}{|l|}{ Encephalopathy — no. (\%)† } \\
\hline None & $191(70)$ & $205(75)$ & $211(77)$ & $190(70)$ & $797(73)$ \\
\hline Grade 1 & $46(17)$ & $38(14)$ & $33(12)$ & $48(18)$ & $165(15)$ \\
\hline Grade 2 & $19(7)$ & $19(7)$ & $16(6)$ & $12(4)$ & $66(6)$ \\
\hline Grade 3 & $5(2)$ & $1(<0.5)$ & $5(2)$ & $7(3)$ & $18(2)$ \\
\hline Grade 4 & 0 & 0 & 0 & 0 & 0 \\
\hline $\begin{array}{l}\text { Sepsis on admission - } \\
\text { no. (\%) }\end{array}$ & $31(11)$ & $27(10)$ & $23(8)$ & $29(11)$ & $110(10)$ \\
\hline $\begin{array}{l}\text { Renal failure on admission - } \\
\text { no. }(\%) \ddagger\end{array}$ & $1(<0.5)$ & 0 & $l(<0.5)$ & 0 & $2(<0.5)$ \\
\hline $\begin{array}{l}\text { Gastrointestinal bleeding } \\
\quad \text { on admission — no. (\%) }\end{array}$ & $16(6)$ & $21(8)$ & $15(5)$ & $15(5)$ & $67(6)$ \\
\hline \multicolumn{6}{|l|}{ Laboratory results } \\
\hline Bilirubin — mg/dl & $17.9 \pm 9.2$ & $17.4 \pm 9.1$ & $17.1 \pm 8.5$ & $17.9 \pm 9.5$ & $17.6 \pm 9.1$ \\
\hline Albumin $-\mathrm{g} /$ liter & $25.6 \pm 6.3$ & $25.2 \pm 6.2$ & $25.1 \pm 5.4$ & $25.3 \pm 6.0$ & $25.3 \pm 6.0$ \\
\hline $\begin{array}{l}\text { Aspartate aminotransferase - } \\
\text { U/liter }\end{array}$ & $143.7 \pm 69.5$ & $133.6 \pm 64.8$ & $134.3 \pm 73.2$ & $143.4 \pm 77.2$ & $138.6 \pm 71.3$ \\
\hline $\begin{array}{l}\text { Alkaline phosphatase - } \\
\text { U/liter }\end{array}$ & $184.7 \pm 86.4$ & $207.7 \pm 113.1$ & $182.4 \pm 85.1$ & $196.1 \pm 98.5$ & $192.7 \pm 96.8$ \\
\hline Creatinine $-\mathrm{mg} / \mathrm{dl}$ & $0.83 \pm 0.43$ & $0.90 \pm 0.52$ & $0.89 \pm 0.55$ & $0.92 \pm 0.58$ & $0.88 \pm 0.53$ \\
\hline White-cell count - per $\mathrm{mm}^{3}$ & $10,100 \pm 5600$ & $10,600 \pm 8100$ & $9900 \pm 5400$ & $9800 \pm 4900$ & $10,100 \pm 6100$ \\
\hline Neutrophils - per $\mathrm{mm}^{3}$ & $7600 \pm 5200$ & $7700 \pm 5200$ & $7400 \pm 4900$ & $7300 \pm 4500$ & $7500 \pm 5000$ \\
\hline Prothrombin time $-\mathrm{sec}$ & $21.1 \pm 5.3$ & $20.8 \pm 5.3$ & $22.1 \pm 6.8$ & $21.1 \pm 5.2$ & $21.3 \pm 5.7$ \\
\hline \multicolumn{6}{|l|}{ Prognostic scores } \\
\hline Discriminant function $\int$ & $61.9 \pm 25.7$ & $60.7 \pm 25.3$ & $65.6 \pm 31.6$ & $62.4 \pm 25.6$ & $62.6 \pm 27.2$ \\
\hline MELD & $20.7 \pm 5.5$ & $21.2 \pm 6.2$ & $21.4 \pm 6.3$ & $21.5 \pm 6.8$ & $21.2 \pm 6.2$ \\
\hline GAHS $\|$ & $8.3 \pm 1.2$ & $8.4 \pm 1.3$ & $8.4 \pm 1.3$ & $8.4 \pm 1.4$ & $8.4 \pm 1.3$ \\
\hline
\end{tabular}

* Plus-minus values are means \pm SD. To convert the values for bilirubin to micromoles per liter, multiply by 17.1. To convert the values for creatinine to micromoles per liter, multiply by 88.4 .

$\dagger$ In patients with encephalopathy, grade 1 indicates mild confusion and impaired attention; grade 2 lethargy, personality change, and inappropriate behavior; grade 3 comatose behavior with responsiveness to verbal or noxious stimuli; and grade 4 coma without responsiveness to verbal or noxious stimuli.

$\nsucceq$ Renal failure was defined as a serum creatinine level greater than $5.7 \mathrm{mg}$ per deciliter or the requirement for renal-support therapy.

$\int$ Discriminant function is calculated as $4.6 \times$ (patient's prothrombin time in seconds - matched control's prothrombin time in seconds) + patient's serum bilirubin level in milligrams per deciliter; a value of 32 or higher indicates severe alcoholic hepatitis that carries an adverse prognosis, with mortality of 20 to $30 \%$ within 1 month after presentation and 30 to $40 \%$ within 6 months after presentation.

I In the Model for End-Stage Liver Disease (MELD), scores range from 6 to 40, with higher scores indicating worse prognosis.

$\|$ The Glasgow alcoholic hepatitis score (GAHS) ranges from 5 to 12, with higher scores indicating worse prognosis.

The New England Journal of Medicine 


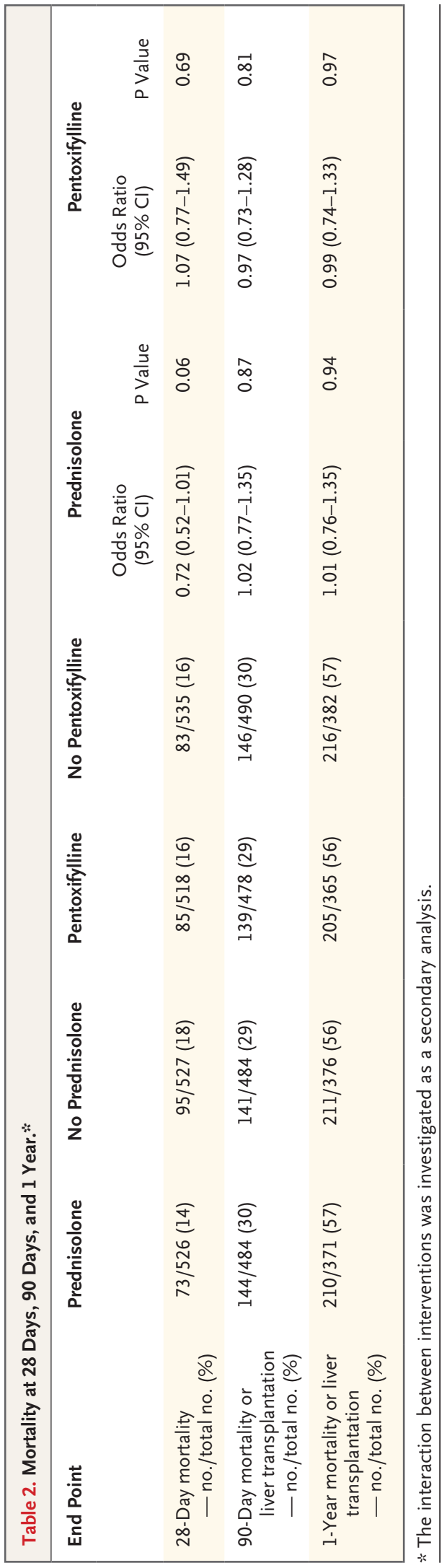

and for survival with prednisolone versus no prednisolone and with pentoxifylline versus no pentoxifylline are provided in Figure 1.

The baseline variables that influenced 28-day mortality in univariate analyses included age, encephalopathy, white-cell count, prothrombin ratio, and serum levels of bilirubin, creatinine, and urea (Table 3). In multivariate analyses, age, encephalopathy, white-cell count, prothrombin ratio, and serum levels of bilirubin, creatinine, and urea remained significant. In a secondary analysis, in which a multivariate logistic-regression model was used that adjusted for these prognostic variables, we found that the odds ratio for 28-day mortality among the patients who received prednisolone, as compared with those who did not, was 0.61 (95\% CI, 0.41 to $0.91 ; \mathrm{P}=0.02$ ) (Table 3). However, the effect of prednisolone on mortality at 90 days (odds ratio, 1.00; 95\% CI, 0.73 to 1.36; $\mathrm{P}=0.98$ ) and at 1 year (odds ratio, 1.01; 95\% CI, 0.74 to $1.39 ; \mathrm{P}=0.94$ ) was nonsignificant.

\section{ADVERSE EVENTS INCLUDING DEATH}

Serious adverse events were reported in $42 \%$ of the patients, with an equal distribution in each of the treatment groups, and $20 \%$ of all serious adverse events resulted in death. Infection occurred in 71 of the 547 patients (13\%) who received prednisolone as compared with 38 of the 545 patients $(7 \%)$ who did not receive prednisolone $(\mathrm{P}=0.002)$. Acute kidney injury occurred in 9 of the 546 patients $(2 \%)$ who received pentoxifylline as compared with 14 of the 546 patients (3\%) who did not receive pentoxifylline (Table 4 , and Table S2 in the Supplementary Appendix).

There were 418 deaths during the trial; 168 (40\%) of these occurred before day $29,28 \%$ occurred between day 28 and day 90 , and $32 \%$ occurred between day 91 and 1 year. Investigators attributed $95 \%$ of the deaths to liver-related causes. Among these causes, infection accounted for $24 \%$ of the deaths, with similar numbers reported for the groups receiving prednisolone and those not receiving prednisolone. The occurrence of gastrointestinal bleeding, sepsis, or renal failure before randomization did not affect mortality during the trial.

\section{DISCUSSION}

Controversy over the use of glucocorticoids in severe alcoholic hepatitis has persisted for many 
Figure 1. Kaplan-Meier Curves Showing Overall Survival According to Study Group.

Kaplan-Meier survival curves show a nonsignificant survival advantage during the first 28 days among patients who received prednisolone as compared with those who did not receive prednisolone (odds ratio, 0.72; $95 \%$ confidence interval [Cl], 0.52 to 1.01 ; $\mathrm{P}=0.06$ ) (Panel $\mathrm{A}$ ). No significant survival advantage was seen for patients who received pentoxifylline as compared with those who did not receive pentoxifylline (odds ratio, $1.07 ; 95 \% \mathrm{Cl}, 0.77$ to $1.49 ; \mathrm{P}=0.69$ ) (Panel B). Survival curves for all four study groups up to 1 year are also shown (Panel C).

years despite the results of meta-analyses of selected trials. ${ }^{5,9}$ In our study, the reduction in 28-day mortality observed among patients treated with prednisolone did not reach the conventional threshold of statistical significance, and no significant differences were observed in 90-day or 12-month outcomes. However, in a secondary analysis that included adjustments for baseline determinants of prognosis, a significant advantage with respect to 28-day mortality was seen with prednisolone. The survival differences may have been a chance finding or may represent a benefit of prednisolone for short-term mortality that did not translate to longer-term benefit.

Although we used the same threshold of disease severity that has been used in most other trials of alcoholic hepatitis (a score of 32 or higher for discriminant function), the 28-day mortality overall in STOPAH was appreciably lower than the 28-day mortality in the trials included in the meta-analysis by Mathurin et al. ${ }^{9}$ However, many of the studies included in the meta-analysis were performed more than 30 years ago, and the mortality reported in two more recently published trials was similar to that reported here. ${ }^{13,15,20}$ There was a lower incidence of infection and acute kidney injury in STOPAH than in some previous trials, a factor that may have contributed to the lower mortality. ${ }^{21}$ A comparison of the baseline characteristics of the patients in our study with those of patients in other trials conducted in the past 4 years shows that in our trial, the mean age was slightly younger and the rate of encephalopathy lower; both characteristics have been consistently shown to influence mortality. ${ }^{15,20}$ However, the bilirubin, creatinine, and albumin levels observed

\section{A Prednisolone vs. No Prednisolone}

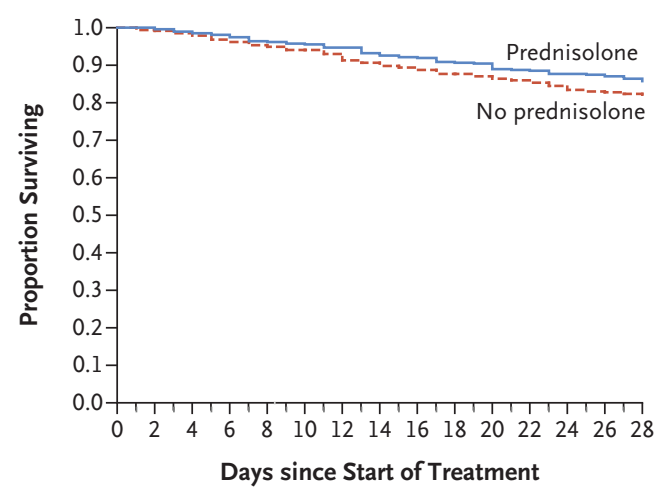

No. at Risk

Prednisolone

No prednisolone

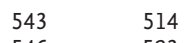

$546 \quad 523$

483

459

427

B Pentoxifylline vs. No Pentoxifylline

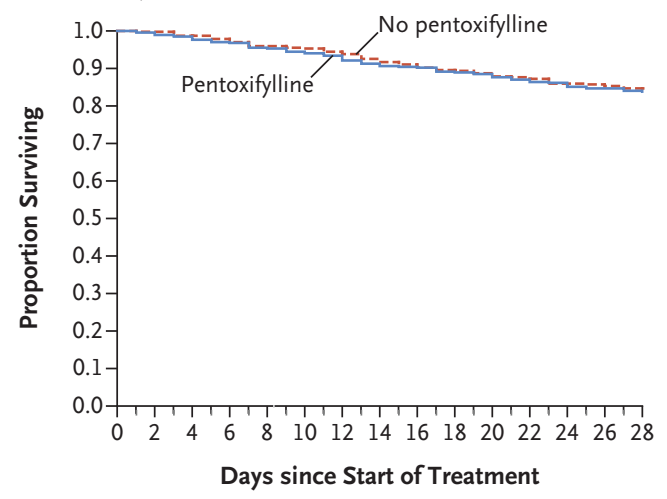

No. at Risk

Pentoxifylline No pentoxifylline

$546 \quad 524$

$543 \quad 513$

497

480

470
457

448

C One-Year Survival in All Groups

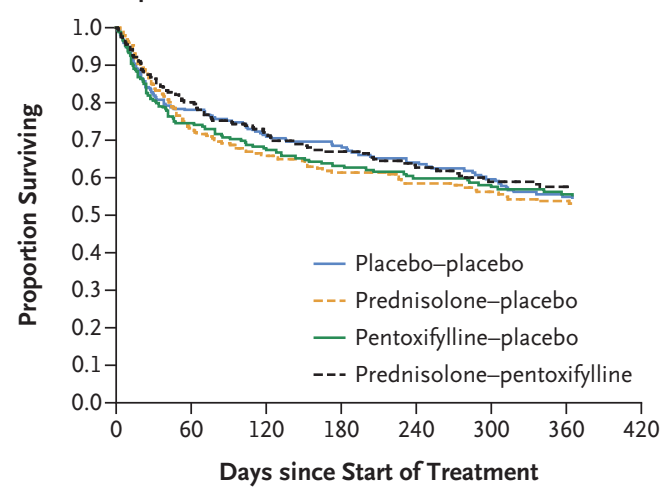

No. at Risk

$\begin{array}{llllllll}\text { Placebo-placebo } & 272 & 199 & 159 & 142 & 121 & 104 & 89\end{array}$

$\begin{array}{llllllrl}\text { Prednisolone-placebo } & 274 & 182 & 139 & 116 & 102 & 91 & 84\end{array}$

$\begin{array}{lllllllll}\text { Pentoxifylline-placebo } & 271 & 178 & 133 & 119 & 104 & 95 & 83\end{array}$

$\begin{array}{llllllll}\text { Prednisolone-pentoxifylline } & 272 & 201 & 157 & 137 & 115 & 101 & 84\end{array}$ 


\begin{tabular}{|c|c|c|c|c|}
\hline \multirow[t]{2}{*}{ Variable } & \multicolumn{2}{|c|}{ Univariate Analysis' } & \multicolumn{2}{|c|}{ Multivariate Analysis: } \\
\hline & $\begin{array}{c}\text { Odds Ratio } \\
(95 \% \mathrm{Cl})\end{array}$ & P Value & $\begin{array}{c}\text { Odds Ratio } \\
(95 \% \mathrm{Cl})\end{array}$ & P Value \\
\hline Prednisolone vs. no prednisolone & & & $0.61(0.41-0.91)$ & 0.02 \\
\hline Pentoxifylline vs. no pentoxifylline & & & $1.10(0.74-1.64)$ & 0.62 \\
\hline Discriminant function & $1.02(1.02-1.03)$ & $<0.001$ & & \\
\hline GAHS & $2.17(1.86-2.53)$ & $<0.001$ & & \\
\hline MELD & $1.15(1.12-1.18)$ & $<0.001$ & & \\
\hline Lille $\mathbb{S}$ & $1.03(1.02-1.03)$ & $<0.001$ & & \\
\hline Prothrombin ratio or INR & $1.38(1.10-1.73)$ & 0.005 & $1.38(1.13-1.69)$ & 0.002 \\
\hline Age & $1.05(1.03-1.07)$ & $<0.001$ & $1.05(1.03-1.07)$ & $<0.001$ \\
\hline White cells & $1.06(1.03-1.08)$ & $<0.001$ & $1.03(1.00-1.06)$ & 0.04 \\
\hline Urea & $1.14(1.10-1.18)$ & $<0.001$ & $1.06(1.01-1.12)$ & 0.01 \\
\hline Creatinine & $3.07(2.32-4.08)$ & $<0.001$ & $1.56(1.05-2.33)$ & 0.03 \\
\hline Pyrexia & $0.66(0.37-1.16)$ & 0.15 & & \\
\hline Hypotension & $1.20(0.79-1.83)$ & 0.39 & & \\
\hline Tachycardia & $1.09(0.71-1.65)$ & 0.70 & & \\
\hline Alcohol intake & $1.00(1.00-1.00)$ & 0.37 & & \\
\hline Albumin & $0.99(0.96-1.02)$ & 0.41 & & \\
\hline Alkaline phosphate & $1.00(1.00-1.00)$ & 0.07 & & \\
\hline Bilirubin & $1.07(1.05-1.09)$ & $<0.001$ & $1.03(1.01-1.06)$ & 0.003 \\
\hline Hepatic encephalopathy & $3.70(2.59-5.29)$ & $<0.001$ & $3.07(2.05-4.60)$ & $<0.001$ \\
\hline
\end{tabular}

* Values reported for all variables were obtained at baseline, and the value for bilirubin was also obtained on day 7 to calculate the Lille score. GAHS denotes Glasgow alcoholic hepatitis score, which ranges from 5 to 12 , with higher scores indicating worse prognosis, INR international normalized ratio, and MELD the Model for End-Stage Liver Disease, in which scores range from 6 to 40, with higher scores indicating worse prognosis.

$\uparrow$ A separate univariate model was fitted for all baseline prognostic factors.

$\checkmark$ Significant variables in the univariate analysis were added to a multivariate model, and backward elimination was applied at the 5\% significance level. Prognostic scores (calculated on the basis of discriminant function, MELD, GAHS and Lille score) were excluded from the multivariate analysis owing to multicolinearity with individual component variables.

$\int$ The Lille score defines the risk of death according to the number of adverse prognostic factors, with higher scores indicating a worse prognosis. We calculated Lille scores on the basis of patient age, albumin level, bilirubin levels (at baseline and on day 7), the prothrombin ratio, and prothrombin time; the formula we used is shown in the protocol.

in patients in STOPAH were similar to those seen in patients in other studies.

The use of liver biopsy to provide histologic confirmation of alcoholic steatohepatitis remains controversial. ${ }^{4,18,19}$ Although many investigators have used histologic confirmation as an entry criterion, it is rarely applied in clinical practice apart from those cases in which the diagnosis is uncertain..$^{19}$ Although it is possible that the diagnosis of alcoholic hepatitis was incorrect in a small number of the patients in our study, when strict clinical criteria are used, it is possible to diagnose the condition with a high degree of accuracy. ${ }^{22}$ Nevertheless, incorrect diagnostic labeling could have reduced the power of the study to detect a therapeutic effect.

A recognized drawback of glucocorticoid use in patients with alcoholic hepatitis is increased susceptibility to infection. ${ }^{21}$ The higher rate of infection among the patients treated with prednisolone was therefore expected, but mortality attributed to infection was similar across the groups, regardless of whether prednisolone was administered. Investigators attributed deaths to infection in 103 of 416 cases (24.8\%), but infection also probably played a role in deaths attributed to other 


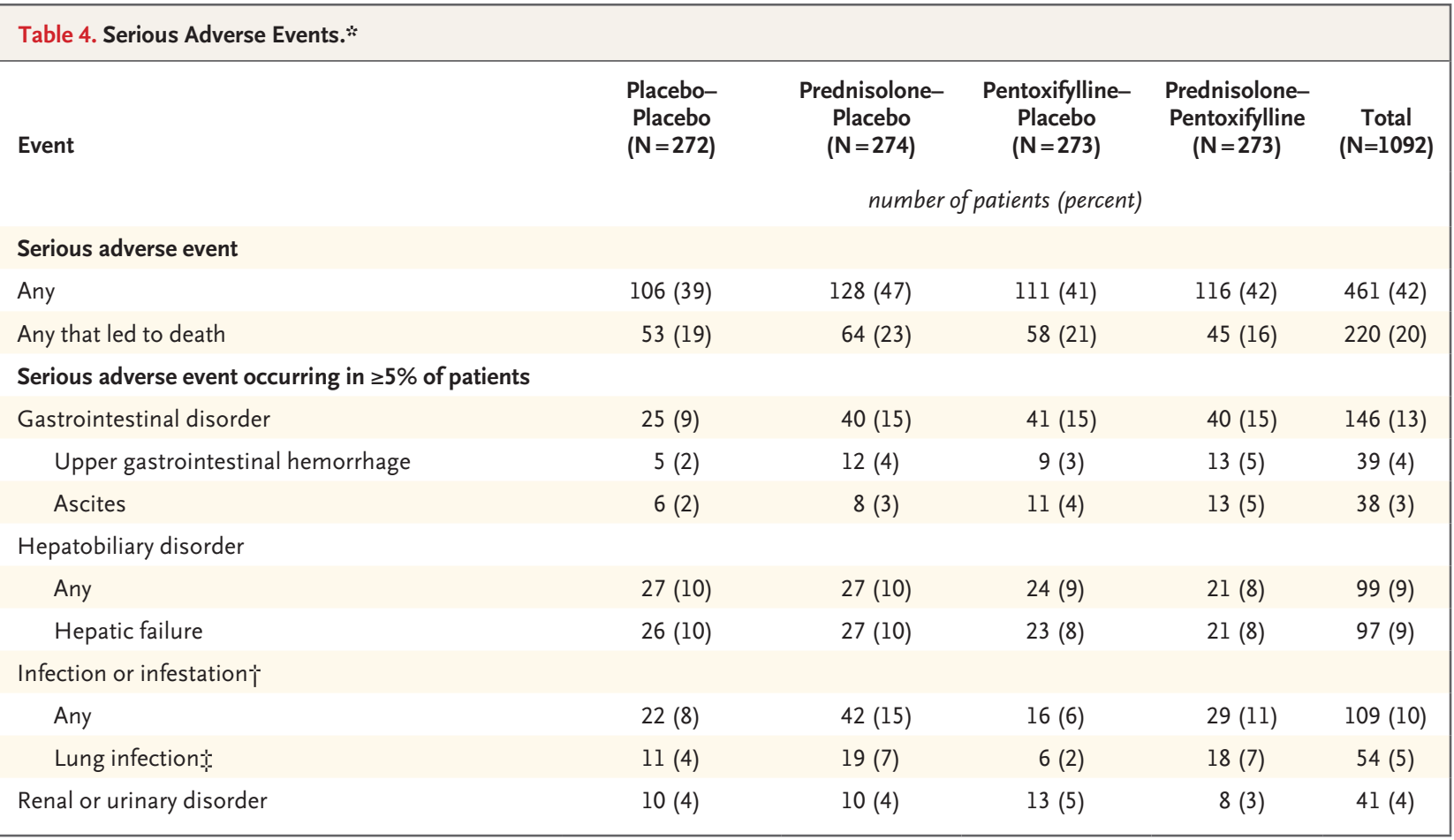

* Adverse events were categorized according to the Common Toxicity Criteria for Adverse Events.

$\dagger P=0.002$ for the comparison of serious adverse events of infection or infestation, which occurred in 71 of 547 (13\%) of the patients who received prednisolone versus 38 of $545(7 \%)$ of the patients who did not receive prednisolone.

$\dagger \mathrm{P}=0.007$ for the comparison of lung infections, which occurred in 37 of 547 (7\%) of the patients who received prednisolone versus 17 of $545(3 \%)$ of the patients who did not receive prednisolone.

causes, such as multiorgan failure. Since infection plays such an important role in the outcome of alcoholic hepatitis, it is worth noting that in a trial published in 2011, the addition of $\mathrm{N}$-acetylcysteine to prednisolone was associated with a reduced rate of infection. ${ }^{20}$

The results of this trial showed that after 28 days, neither prednisolone nor pentoxifylline influenced mortality. Furthermore, the cumulative mortality at 90 days and at 1 year in this group of patients is alarming. Self-reported alcohol consumption revealed complete abstinence in $37 \%$ of the patients at 1 year of follow-up. However, data on alcohol consumption are difficult to collect, and this fact is reflected in the high proportion of missing data. No matter what the exact figures are, more clearly needs to be done to prevent recidivism in this group of patients.
In summary, in the STOPAH trial, pentoxifylline did not improve outcomes in patients with alcoholic hepatitis. The findings suggest that the administration of $40 \mathrm{mg}$ of prednisolone daily for 1 month may have a beneficial effect on shortterm mortality but not on the medium-term or long-term outcome of alcoholic hepatitis.

Supported by a grant $(0814$ 44) from the National Institute for Health Research (NIHR) Health Technology Assessment program.

Dr. Thursz reports receiving lecture fees and consulting fees from Gilead, Bristol-Myers Squibb, AbbVie, and Abbott; and Dr. Allison, receiving consulting fees from Norgine. No other potential conflict of interest relevant to this article was reported.

Disclosure forms provided by the authors are available with the full text of this article at NEJM.org.

We thank the NIHR Clinical Research Network for providing support for research nurses and the Imperial College Biomedical Research Centre, Southampton Clinical Trials Unit, for management of the trial.

\section{APPENDIX}

From Imperial College (M.R.T., N.V.), King's College Hospital (J.O.), and the Royal Free Hospital (D.P.), London, Royal Liverpool Hospital (P. Richardson) and Aintree Hospital (S.H.), Liverpool, Addenbrookes Hospital, Cambridge (M.A.), Derby Royal Hospital, Derby (A.A.), Southampton Clinical Trials Unit, University of Southampton (M.B., N.D., J.M., I.R., P. Roderick, L.S.), and University Hospital Southampton NHS Foundation Trust (M.W.), Southampton, Faculty of Medical Sciences, Newcastle University (C.P.D.), and 
Newcastle upon Tyne Hospitals NHS Foundation Trust (S.M.), Newcastle upon Tyne, Sheffield Teaching Hospitals Foundation Trust, Sheffield (D.G.), Edinburgh Royal Infirmary, Edinburgh (A. MacGilchrist), Leicester Royal Infirmary, Leicester (A.G.), Bristol Royal Infirmary, Bristol (A. McCune), Nottingham University Hospitals NHS Trust and National Institute for Health Research Biomedical Research Unit, Queens Medical Centre, Nottingham (S.R.), and the Glasgow Royal Infirmary, Glasgow (E.H.F.) — all in the United Kingdom.

REFERENCES

1. Lucey MR, Mathurin P, Morgan TR. Alcoholic hepatitis. N Engl J Med 2009; 360:2758-69.

2. Maddrey WC, Boitnott JK, Bedine MS, Weber FL Jr, Mezey E, White RI Jr. Corticosteroid therapy of alcoholic hepatitis. Gastroenterology 1978;75:193-9.

3. European Association for the Study of Liver. EASL clinical practical guidelines: management of alcoholic liver disease. J Hepatol 2012;57:399-420.

4. O'Shea RS, Dasarathy S, McCullough AJ. Alcoholic liver disease. Hepatology 2010;51:307-28.

5. Rambaldi A, Saconato HH, Christensen E, Thorlund K, Wetterslev J, Gluud C. Systematic review: glucocorticosteroids for alcoholic hepatitis - a Cochrane Hepato-Biliary Group systematic review with meta-analyses and trial sequential analyses of randomized clinical trials. Aliment Pharmacol Ther 2008; 27:1167-78.

6. Mendenhall CL, Anderson S, GarciaPont P, et al. Short-term and long-term survival in patients with alcoholic hepatitis treated with oxandrolone and prednisolone. N Engl J Med 1984;311:1464-70. 7. Ramond MJ, Poynard T, Rueff B, et al. A randomized trial of prednisolone in patients with severe alcoholic hepatitis. N Engl J Med 1992;326:507-12

8. Mathurin P, Duchatelle V, Ramond MJ, et al. Survival and prognostic factors in patients with severe alcoholic hepatitis treated with prednisolone. Gastroenterology 1996;110:1847-53.

9. Mathurin P, O'Grady J, Carithers RL, et al. Corticosteroids improve short-term survival in patients with severe alcoholic hepatitis: meta-analysis of individual patient data. Gut 2011;60:255-60.

10. Akriviadis E, Botla R, Briggs W, Han S, Reynolds T, Shakil O. Pentoxifylline improves short-term survival in severe acute alcoholic hepatitis: a double-blind placebo-controlled trial. Gastroenterology 2000;119:1637-48.

11. Whitfield K, Rambaldi A, Wetterslev J, Gluud C. Pentoxifylline for alcoholic hepatitis. Cochrane Database Syst Rev 2009;4:CD007339.

12. Parker R, Armstrong MJ, Corbett C, Rowe IA, Houlihan DD. Systematic review: pentoxifylline for the treatment of severe alcoholic hepatitis. Aliment Pharmacol Ther 2013;37:845-54.

13. Park SH, Kim DJ, Kim YS, et al. Pentoxifylline vs. corticosteroid to treat severe alcoholic hepatitis: a randomised, noninferiority, open trial. J Hepatol 2014;61: 792-8.

14. De BK, Gangopadhyay S, Dutta D Baksi SD, Pani A, Ghosh P. Pentoxifylline versus prednisolone for severe alcoholic hepatitis: a randomized controlled trial World J Gastroenterol 2009;15:1613-9.

15. Mathurin P, Louvet A, Duhamel A, et al. Prednisolone with vs without pentoxifylline and survival of patients with severe alcoholic hepatitis: a randomized clinical trial. JAMA 2013;310:1033-41.

16. Sidhu SS, Goyal O, Singla P, et al. Corticosteroid plus pentoxifylline is not better than corticosteroid alone for improving survival in severe alcoholic hepatitis (COPE trial). Dig Dis Sci 2012;57: 1664-71.

17. Forrest E, Mellor J, Stanton L, et al. Steroids or Pentoxifylline for Alcoholic Hepatitis (STOPAH): study protocol for a randomised controlled trial. Trials 2013; 14:262.

18. Potts JR, Goubet S, Heneghan MA, Verma S. Determinants of long-term outcome in severe alcoholic hepatitis. Aliment Pharmacol Ther 2013;38:584-95.

19. Singal AK, Salameh H, Singal A, et al. Management practices of hepatitis $C$ virus infected alcoholic hepatitis patients: A survey of physicians. World J Gastrointest Pharmacol Ther 2013;4:16-22.

20. Nguyen-Khac E, Thevenot T, Piquet MA, et al. Glucocorticoids plus N-acetylcysteine in severe alcoholic hepatitis. N Engl J Med 2011;365:1781-9.

21. Louvet A, Wartel F, Castel H, et al. Infection in patients with severe alcoholic hepatitis treated with steroids: early response to therapy is the key factor. Gastroenterology 2009;137:541-8.

22. Forrest EH, Gleeson D. Is a liver biopsy necessary in alcoholic hepatitis? J Hepatol 2012;56:1427-8.

Copyright (c) 2015 Massachusetts Medical Society. 